\title{
Neue Leitlinien zum Vorhofflimmern
}

\section{Dronedaron mit Klasse-IA-Empfehlung}

\begin{abstract}
IDI Nach den aktualisierten Leitlinien zum Management von Vorhofflimmern ist die Auswahl an Antiarrhythmika um eine wichtige Substanz erweitert worden: Dronedaron hat nun zur Aufrechterhaltung des Sinusrhythmus bei paroxysmalem und persistierendem Vorhofflimmern eine KlasseIA-Empfehlung erhalten. Das ist eine wichtige Nachricht für die medizinische Praxis, denn Vorhofflimmern ist häufig: 4,3 Millionen Patienten leiden darunter in Europa, ein Drittel aller stationären Einweisungen wegen Arrhythmien gehen auf seine Kosten.
\end{abstract}

Wesentlich für die Bewertung von Dronedaron war die ATHENA-Studie mit über 7000 Patienten mit Vorhofflimmern (VHF) bzw. -flattern, eine randomisierte, placebokontrollierte 30-Monate-Studie. Tod und Hospitalisationen wegen VHF, der primäre Endpunkt, wurde signifikant gesenkt. Wie Studienleiter Stephan Hohnloser, Frankfurt, betonte, sind solche Hospitalisationen nicht nur kostensteigernd, sondern ein Surrogatmarker für die Mortalität. Mit ATHENA wurde erstmalig die Bedeutung der antiarrhythmischen Therapie auf Hospitalisierung und Tod belegt. In den Leitlinien wird nun zum ersten Mal auf die Notwendigkeit verwiesen, stationäre Einweisungen möglichst zu vermeiden.

Die IA-Einstufung von Dronedaron bezieht sich auf das paroxysmale und persistierende VHF. Nicht so nachhaltig ist die Empfehlung im Falle von VHF bei chronischer (Stadien III/IV) oder instabiler Herzinsuffizienz (Dekompensation im vorausgehenden Monat): IIIB. Dagegen kann Dronedaron sicher gegeben werden bei Patienten mit akutem Koronarsyndrom, chronischer Angina pectoris, hypertensiver Herzerkrankung und stabiler leichter Herzinsuffizienz (Stadien I und II).

(J.A.)II

\section{Kommentar}

\section{Überzogen: Strauer kassiert für Vorab-Publikation von STAR die rote Karte}

Zunächst wohlwollender Applaus, dann die Abstrafung: Prof. Bodo-Eckehard Strauer, Studienleiter der STAR Heart Study, wusste nicht wie ihm geschah. Er durfte als erster Redner in der ersten Hot-Line-Session die Ergebnisse der intrakoronaren Stammzellbehandlung an 191 Patienten mit chronischer Herzinsuffizienz aufgrund ischämischer Kardiomyopathie vortragen eine Analyse nach im Mittel knapp fünf Jahren. Der Diskussionsredner F. Fernandez-Aviles, Madrid, sah in STAR eine Bestätigung, dass der klinische Nutzen unbestritten ist, auch wenn die Wirkmechanismen ungeklärt sind und die Studie nicht randomisiert war.

\section{Eklat um eine Formalie}

Der Eklat hatte mit den Ergebnissen auch nichts zu tun. Es ging um eine reine Formalie: Die Ergebnisse waren kurz vor Kongressbeginn bereits in einem Journal der European Society of Cardiology (ESC) erschienen, was vom HotLine-Kommittee untersagt ist. Vor wenigen Jahren wäre deswegen wohl noch niemand aufgebraust. Strauer, mit dem Vorwurf konfrontiert, wand sich

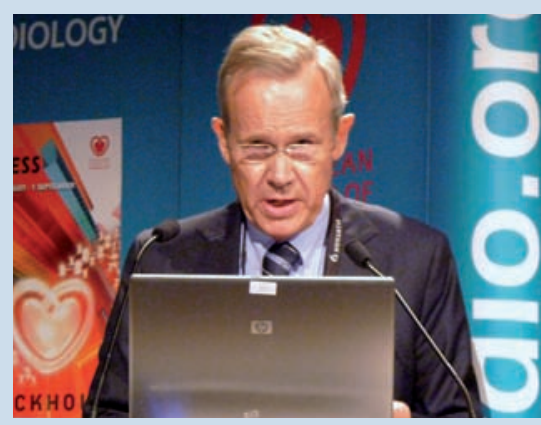

Bei den ESC-Granden in Ungnade gefallen: Prof. Bodo-Eckehard Strauer.

ein wenig und meinte, er habe nicht damit gerechnet, dass die Publikation so rasch erfolgt. Wie auch immer, die ESC-Präsidialen ließen umgehend im Presseraum ein Abstraf-Statement an die Wand pinnen, es bezichtigte Strauer, die Regeln der Hotline-Sessions gebrochen zu haben, ein strafbewehrtes Vergehen. Das Urteil: Die ESC wird in den nächsten zwei Jahren von Strauer kein Abstract mehr annehmen.

Überzogen, unfair und selbstgefällig Kein Zweifel, Strauer hat gegen eine Regel verstoßen, aber rechtfertigt dies zum einen die öffentliche Demütigung via Presseerklärung und zum zweiten ein Publikationsverbot, das einer Ächtung in der wissenschaftlichen Community gleichkommt? Und muss sich die ESC nicht auch an die eigene Nase fassen, weil die Arbeit in einem ihrer Publikationsorgane erschienen ist? Lesen diese kardiologischen Alphamännchen etwa ihre eigenen Blätter nicht? Natürlich steckt hinter dieser Strafaktion auch pure Selbstgefälligkeit. Die ESC-Vorstände - darunter kein Deutscher - wollen ihre Macht demonstrieren, vor allem gegenüber den amerikanischen Kollegen, die auf solche Verstöße schon seit Jahren sehr allergisch reagieren. „Wir können auch streng sein", heißt die Botschaft. So gesehen war Strauer nur ein Bauernopfer. Es sollte künftig etwas fairer und europäischer, d.h. Kompromisse suchend, zugehen. Man muss doch wegen eines solchen Lapsus nicht gleich Kriegstänze aufführen. Die gelbe Karte hätte genügt. Im Fußball wird die rote Karte nur gegeben, wenn ein grobes Foul vorliegt, das die Gesundheit eines Mitspielers gefährdet. Den ESC-Granden würde ein Coaching in Sachen Fairness nicht schaden.

Dr. med. Jochen Aumiller II 